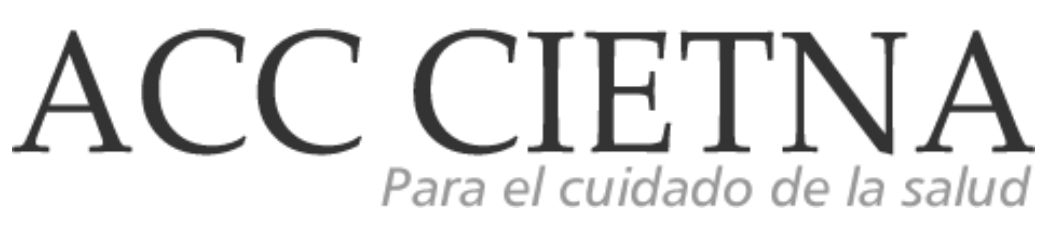

https://doi.org/10.35383/cietna.v8i2.652

e-ISSN: $2309-8570$

Universidad Católica Santo Toribio de Mogrovejo

\title{
Validación de un instrumento para determinar conductas sedentarias en universitarios
}

\section{Validation of an instrument to determine sedentary behaviors in university students}

\author{
Julio César Méndez-Ávila *, 1, a \\ julio_uaq@hotmail.com \\ https://orcid.org/0000-0002-4059-2288
}

José Miguel Silva-Llaca ${ }^{1, b}$

msll.uaq@gmail.com

https://orcid.org/0000-0002-1527-0033

\author{
Alan Ornelas-Murrieta ${ }^{1, c}$ \\ alan96_ags@hotmail.com \\ https://orcid.org/0000-0002-9970-9735
}

María Antonieta Mendoza-Ayala 1, d
marianjom@gmail.com
https://orcid.org/0000-0001-7907-5045

Arely Guadalupe Morales-Hernández ${ }^{1 \text {, e }}$ arely.morales@uaq.mx

https://orcid.org/0000-0002-7420-7210

\author{
* Autor corresponsal \\ 1 Facultad de Enfermería, Universidad Autónoma de \\ Querétaro, Querétaro, México \\ a Jefe de Investigación y Postgrado \\ b Coordinador de Maestría en Ciencias del Deporte \\ c Licenciado en Educación Física y Ciencias del Deporte \\ d Docente \\ e Coordinador de Maestría en Ciencias de la \\ Rehabilitación en el Movimiento Humano
}

Fechas importantes

Recibido: 2021-10-13

Aceptado: 2021-11-15

Publicado online: 2021-12-17

\begin{abstract}
Resumen
Objetivo: Validar un instrumento de recolección de datos para determinar conductas sedentarias en universitarios. Método: La investigación se condujo a través de un diseño de tipo mixto de carácter exploratorio secuencial (DEXPLOS), con una etapa cualitativa y una cuantitativa. Para la etapa cualitativa se utilizó la técnica de grupos focales donde participaron 24 estudiantes con una guía de entrevista semiestructurada que fue sometida a una revisión de cinco expertos y se analizó con el programa ATLAS TI 8. Posteriormente en la etapa cuantitativa se realizó un muestreo por conglomerados dividendo a la población de $1^{\circ}$ a $10^{\circ}$ semestre, obteniendo una muestra de 200 estudiantes de las carreras de Enfermería, Fisioterapia y Educación Física y Ciencias del Deporte. Partiendo de ello se elaboró un cuestionario propio con una escala de Likert con 7 opciones de respuesta y para el análisis de datos cuantitativos se utilizó el programa SPSS versión 25. Resultados principales: Para la etapa cualitativa, se encontraron tres categorías que engloban contextualmente las actividades realizadas en la cotidianidad por los universitarios: estilo de vida, actividad física y tiempo libre. Para la etapa cuantitativa, el instrumento tuvo una validación del coeficiente de Alpha de Cronbach de 0.780 y Coeficiente de Guttman de 0.751 . Conclusión general: El cuestionario propuesto en este estudio es adecuado para estimar de manera objetiva y contextualizada conductas y hábitos en estudiantes universitarios queretanos ya que tiene una fiabilidad por encima del límite establecido.
\end{abstract}

Palabras clave: Familia, sedentarismo, estudiantes universitarios 


\begin{abstract}
Objective: To validate a data collection instrument to determine sedentary behaviors in university students. Method: The research was conducted through a sequential exploratory mixed type design (DEXPLOS), with a qualitative and a quantitative stage. For the qualitative stage, the focus group technique was used in which 24 students participated with a semi-structured interview guide that was subjected to a review by five experts and was analyzed with the ATLAS TI 8 program. Later, in the quantitative stage, a sampling by conglomerates dividing the population from 1st to 10th semester, obtaining a sample of 200 students from the careers of Nursing, Physiotherapy and Physical Education and Sports Sciences. Based on this, an own questionnaire was developed with a Likert scale with 7 response options and the SPSS version 25 program was used for the analysis of quantitative data. Main results: For the qualitative stage, three categories were found that contextually encompass the activities carried out in daily life by university students: lifestyle, physical activity, and free time. For the quantitative stage, the instrument had a validation of the Cronbach's Alpha coefficient of 0.780 and Guttman's coefficient of 0.751 . General conclusion: The questionnaire proposed in this study is adequate to estimate in an objective and contextualized way behaviors and habits in Queretaro university students since it has a reliability above the established limit.
\end{abstract}

Keywords: Validation, sedentary lifestyle, university students

\title{
Introducción
}

La actividad física de manera regular es determinante para la prevención y tratamiento de enfermedades no transmisibles (ENT) como la diabetes, cáncer y enfermedades cardiovasculares y proporciona amplios beneficios físicos y psicológicos. Estudios revelan que a nivel mundial el $81 \%$ de los jóvenes ${ }^{(1)}$ no cumplen las recomendaciones mundiales que la Organización Mundial de la Salud (OMS) instauró desde el 2010, sobre la cantidad y calidad necesarias para la actividad física (2).

A nivel nacional, según datos de la Encuesta Nacional sobre Uso del Tiempo (ENUT) 2019, el $77 \%$ de los adolescentes y jóvenes mexicanos no realizan deporte o ejercicio físico, además de que el tiempo dedicado frente a una pantalla es tres veces mayor que a las actividades físico-deportivas (3). Asimismo, datos a nivel municipal en Querétaro revelan que el $40.27 \%$ de los estudiantes no realizan la actividad física recomendada y $45.17 \%$ pasa en promedio cuatro horas diarias en las redes sociales ${ }^{(4)}$.

Al respecto es importante mencionar que la mayoría de los estudios que miden niveles de actividad física utilizan el IPAQ y GPAQ para tipificar el tiempo en movimiento y el tiempo sedentario ${ }^{(5)}$. Sin embargo, dichos instrumentos se ven limitados y alejados de la realidad y del contexto inmediato de una población universitaria y es posible que tengan una validez de criterio pobre ${ }^{(6)}$. Los estilos de vida actualmente en los universitarios se han visto modificados por distintas variables, las cargas académicas, la necesidad de trabajar, la falta de hábitos de actividad física o ejercicio físico.

En este sentido, estudios realizados en poblaciones universitarias revelan una relación positiva entre el uso excesivo del celular y el comportamiento sedentario. Aunque, por otra parte, se distingue el uso de herramientas tecnologías para realizar actividad física a través de las aplicaciones que estimulan una vida más activa (7). En virtud de lo anterior, se resalta la importancia y pertinencia de implementar intervenciones en los universitarios debido a los problemas de sedentarismo que la vida académica estudiantil conlleva ${ }^{(8)}$. Por ello, el objetivo de la investigación radica en ofrecer una propuesta valida, contextualizada y pertinente para recolectar datos que permitan determinar conductas sedentarias en estudiantes universitarios queretanos. 


\section{Material y métodos}

La investigación se condujo a través de un diseño de tipo mixto de carácter exploratorio secuencial (DEXPLOS), ya que para la validación de un instrumento sobre conductas sedentarias en estudiantes universitarios inicialmente se realizó un abordaje cualitativo con su recolección-análisis de datos y en función de los resultados obtenidos y analizados se desarrolló una fase cuantitativa ${ }^{(9)}$.

De tal manera, en atención del enfoque mixto y de la profundidad que esta investigación sugiere, primero se consideró realizar un abordaje cualitativo con el objetivo de comprender el pensar, sentir y vivir de los estudiantes universitarios en relación con los hábitos sedentarios a través de la recopilación discursiva de los actores de las tres licenciaturas de la Facultad de Enfermería de la Universidad Autónoma de Querétaro (UAQ-México). De tal forma, se utilizaron grupos focales como técnica de recolección de datos y para cumplir los criterios de homogeneidad y heterogeneidad que esta técnica necesita, se realizaron cuatro grupos donde se integraron equitativamente estudiantes de cada una de las licenciaturas. El primero fue de seis estudiantes inscritos en segundo semestre, el segundo grupo tuvo seis estudiantes inscritos en cuarto semestre, el tercero integró seis estudiantes cursando el sexto semestre y finalmente, el cuarto grupo focal estuvo constituido por seis estudiantes inscritos en el octavo semestre.

Para la recolección de datos cualitativa se utilizó una guía de entrevista semiestructurada que estuvo constituida por 12 preguntas y fue sometida a una revisión de cinco expertos para su validación. Se hizo la prueba piloto con cuatro estudiantes de distintas licenciaturas y semestres de la universidad. Posteriormente, durante el año 2019 en las instalaciones de la Facultad de Enfermería de la UAQ se realizaron las entrevistas a los 24 estudiantes por invitación con una duración aproximada de 50 a 70 minutos por grupo focal. Para el análisis de los datos cualitativos, posterior a la transcripción del material discursivo obtenido en los grupos focales, se realizó un tratamiento pertinente a través del programa Atlas Ti mediante el sistema de codificación, ordenación y clasificación en función de las categorías inductivas principales, secundarias y sus indicadores obtenidos.

Posteriormente con los datos arrojados por el estudio cualitativo, se realizó un estudio de tipo cuantitativo con una población de 600 estudiantes de nivel licenciatura inscritos en la Facultad de Enfermería de la UAQ-México durante el año 2020. Para ello, se llevó a cabo un muestreo por conglomerados que dividió a la población total por semestres $\left(1^{\circ}\right.$ a $\left.10^{\circ}\right)$ obteniendo una muestra de 200 estudiantes de las tres licenciaturas integradas en la Facultad.

El instrumento cuantitativo se realizó con base en los estudios revisados y el análisis de los cuestionarios aplicados dentro de estos estudios donde destacan el IPAQ ${ }^{(10)}$ y el GPAQ ${ }^{(11)}$. Partiendo de ello se elaboró un cuestionario con una escala de Likert con 7 opciones de respuesta, inicialmente el cuestionario se conformó de 27 preguntas y se aplicó a 200 universitarios para su evaluación. Esta revisión brindó información importante para estructurar cada ítem, observando que a los jóvenes se les complica recordar las actividades que realizan durante la semana y que para ellos es más significativo recordar sus hábitos entre semana y fines de semana. Así, para la validación final del cuestionario se utilizó el programa SPSS versión 25 mediante el coeficiente de fiabilidad de Alpha de Cronbach y Guttman.

A todos los participantes se les informó sobre el objetivo de la investigación, aceptaron de manera voluntaria firmando el consentimiento informado que atiende los principios de la Declaración de Helsinki, Código de Nuremberg, Reglamento de la Ley General de Salud en 
materia de Investigación. El proyecto fue aprobado por el Sub-Comité de Investigación y Comité de Bioética de la Facultad de Enfermería UAQ.

\section{Resultados y discusión}

En los resultados emergieron tres grandes categorías que engloban contextualmente las actividades realizadas en la cotidianidad por estudiantes de las tres licenciaturas: C1 Estilo de vida (ver tabla $\mathrm{N}^{\circ} 01$ ).; C2 Actividad Física (ver tabla $\mathrm{N}^{\circ} 02$ ).; y C3 Tiempo Libre (ver tabla $\mathrm{N}^{\circ}$ 03).

Tabla N॰ 01

\section{Categoría Estilos de vida}

\begin{tabular}{|l|l|}
\hline C1 - Estilo de vida & \multicolumn{2}{|l|}{ Indicadores } \\
\hline \multirow{4}{*}{ Dúa cotidiano } & Estudios \\
\cline { 2 - 2 } & Prácticas \\
\cline { 2 - 2 } & Despertar temprano y dormir tarde \\
\cline { 2 - 2 } & Actividad física o tiempo libre \\
\hline \multirow{4}{*}{ Actividades prioritarias } & Escuela y tareas \\
\cline { 2 - 2 } & Comer \\
\cline { 2 - 2 } & Horas de sueño \\
\cline { 2 - 2 } & Familia y tiempo libre \\
\hline \multirow{5}{*}{ Sedentarismo } & Hacer por obligación \\
\cline { 2 - 2 } & Ser solitario \\
\cline { 2 - 2 } & Ser rutinario \\
\cline { 2 - 2 } & No hacer ejercicio \\
\cline { 2 - 2 } & No ser productivo \\
\hline
\end{tabular}

\section{Elaboración propia}

La tabla $\mathrm{N}^{\circ} 01$ da cuenta de la Categoría Estilo de vida que se configura a través de las relaciones conceptuales de red halladas y determinadas por tres subcategorías. Así, en lo referente al día cotidiano el estilo de vida de los universitarios puede estimarse en virtud del tiempo dedicado a la jornada escolar dentro de la institución y fuera de ella bajo el modelo de las prácticas curriculares. Además de la tensión para decidir entre dedicar tiempo para el ocio o para la actividad física y la dificultad para satisfacer un adecuado ciclo de sueño. Esto es congruente con otros estudios que revelan que durante el sueño se desarrollan importantes funciones del organismo y de la actividad cerebral y se recomienda que los jóvenes deben descansar un promedio de 6 a 8 horas, es una realidad que muchos jóvenes padecen trastornos de sueño ${ }^{(12)}$.

Por otra parte, se distingue la subcategoría de las actividades prioritarias, es decir, aquello que los universitarios estiman fundamental e imperativo en su día cotidiano. En ella, los indicadores para su estimación contemplan el tiempo destinado para la asistencia al centro escolar y las tareas, así como los horarios de comida, sueño y lo destinado para la convivencia con los familiares y el tiempo libre personal.

Por último, bajo el reconocimiento de los bajos niveles de activación física que la naturaleza y demanda de sus ofertas profesionales les permiten, emerge la subcategoría del sedentarismo. En ella, los estudiantes reconocen como no esencial el tiempo destinado a la actividad física en contraposición con los indicadores de la subcategoría de actividades prioritarias. De tal forma, la noción sedentaria se puede estimar a través de indicadores como la sensación de 
estar solo o vivir en una rutina constante y por tanto asumirse como no productivo, así como no realizar ejercicio físico alguno durante el día a día.

Tabla $\mathbf{N}^{\circ} 02$

Categoría Actividad Física

\begin{tabular}{|l|l|}
\hline C2 - Actividad Física & Indicadores \\
\hline Subcategorías & Mucho tiempo sentado \\
\hline \multirow{2}{*}{ Jornada Universitaria } & Actividades de la profesión \\
\hline \multirow{2}{*}{ Sedentarismo/ Actividad Física universitaria } & Mi carrera propicia el sedentarismo \\
\cline { 2 - 2 } & Mi carrera me mantiene activo \\
\hline
\end{tabular}

\section{Elaboración propia}

La tabla $\mathrm{N}^{\circ} 02$ ofrece información relevante respecto de la categoría Actividad Física, configurada en las relaciones encontradas durante la codificación discursiva con dos subcategorías. En este sentido, los universitarios se significan en cuanto a la Actividad Física en torno a la actividad principal de su día representada por la jornada escolar. Así, es plausible aproximarse tanto a su calidad como cantidad mediante indicadores como el tiempo que se mantienen sentados durante su día académico y que las actividades propias de la oferta educativa que no permiten activarse físicamente fuera del espacio escolar. No obstante, también se distingue una tensión paradigmática, ya que los universitarios conciben que cursar una carrera del área de la salud representa para ellos una disminución significativa en sus niveles de Actividad Física regular y aunque algunos la realizan en asignaturas destinadas a ello, a medida que avanzan en la oferta educativa tales espacios desaparecen y se incrementa la carga académica. Es una realidad que actualmente ha existido una disminución de la actividad física, actualmente los patrones de conducta de los universitarios van mayormente enfocados a conductas sedentarias ${ }^{(13)}$.

\begin{tabular}{|c|c|}
\hline \multicolumn{2}{|c|}{$\begin{array}{l}\text { Tabla } N^{\circ} 03 \\
\text { Tiempo libre }\end{array}$} \\
\hline C3 - Tiempo Libre & \\
\hline Subcategorías & Indicadores \\
\hline \multirow{5}{*}{ Uso de tiempo libre } & Familia \\
\hline & Fiestas, amigos \\
\hline & Estudiar \\
\hline & No hay tiempo libre \\
\hline & Actividades de ocio \\
\hline Redes sociales & Uso prolongado durante todo el día \\
\hline \multirow{3}{*}{ Sedentarismo/actividad física } & No tiempo libre \\
\hline & Descansar es prioridad \\
\hline & Estar con familia \\
\hline
\end{tabular}

\section{Elaboración propia}

La tabla $N^{\circ} 03$ proporciona información pertinente sobre la configuración de la categoría Tiempo Libre y sus respectivas subcategorías. En primer lugar, es destacable que la demanda tiempo-esfuerzo que implican los programas educativos es amplia y genera complicaciones para conciliar la jornada escolar, las prácticas profesionales, el servicio social y las actividades extraescolares correspondientes como tareas y labores de investigación, dejando poco o nulo espacio para considerarlo tiempo libre. Por lo anterior, cuando existe, la noción de tiempo libre es vital y se puede tener una estimación de ella con indicadores que revelan su uso para 
destinarle a la familia, a las fiestas, las amistades o actividades de ocio personal como mirar televisión. A diferencia del estudio de Práxedes y Col donde los programas relacionados al área de la salud promueven realizar actividad física en el tiempo libre ${ }^{(14)}$.

Además, resulta importante reconocer la trascendencia que tienen las redes sociales en las relaciones de los universitarios ya que superan la barrera de la jornada escolar y tiempo libre, es decir, su uso es indistinto durante el día, lo que genera una acumulación excesiva y significativa de tiempo destinado a ellas.

Por último, en relación de concordancia con los conceptos entretejidos en la red de la codificación, la correspondencia de actividad física y actividades sedentarias evidencia poco o nulo tiempo libre para dedicarle a uno u otro. No obstante, cuando se reconoce la posibilidad de tenerlo, existen bajos niveles de ejercicio físico al anteponer el uso de ese tiempo libre para el descanso físico-mental y para la convivencia con la familia-amigos.

Luego de analizar los resultados cualitativos se procedió a elaborar el instrumento de recolección de datos, que está integrado por 3 factores: actividad física, estilos de vida, tiempo libre y 10 preguntas sociodemográficas (semestre, turno, tipo de institución, carrera, edad, genero, trabajo, con quién vive y la escolaridad de los padres).

Para la validación del instrumento se examinaron los siguientes aspectos: validez de contenido, validez de constructo y fiabilidad del cuestionario.

Validez de contenido: para la validación del cuestionario, éste se aplicó a una muestra piloto inicial de 105 estudiantes de distintas licenciaturas de la Universidad Autónoma de Querétaro (UAQ-México). Antes de contestar se les dio la indicación de leer cuidadosamente y donde existieran dudas lo manifestaran, lo anterior tenía la finalidad de revisar la redacción. Los resultados fueron los siguientes: se modificaron las preguntas $1,5,7,9,11$ y 13 porque eran variables que no consideraban que el tiempo dedicado a realizar actividad física y uso de las redes sociales cambia significativamente entre semana y los fines de semana. En las preguntas sociodemográficas se agregó la pregunta 7 ya que una variable que determina el uso del tiempo libre es si trabaja el estudiante. Después de realizados los cambios se aplicó a la muestra definitiva de 200 estudiantes (Ver tabla No 04).

Validez de constructo: considerando que todas las variables que integran el constructo son nominales y ordinales, se tomó la decisión de utilizar las técnicas estadísticas: ANOVA, Ji Cuadrada y Análisis factorial. Para ver la dependencia de las variables nominales, con las ordinales y continuas. Se realizó la validez del constructo con el tamaño de la muestra de 200 cuestionarios.

Tabla $N^{\circ} 04$

Valor de alfa de Cronbach por cada ítem

\begin{tabular}{|c|c|}
\hline Pregunta & Alpha de Cronbach \\
\hline P1 & .761 \\
\hline P2 & .772 \\
\hline P3 & .777 \\
\hline P4 & .778 \\
\hline P5 & .763 \\
\hline P6 & .754 \\
\hline P7 & .779 \\
\hline P8 & .777 \\
\hline
\end{tabular}




\begin{tabular}{|c|l|}
\hline P9 & .772 \\
\hline P10 & .761 \\
\hline P11 & .767 \\
\hline P12 & .772 \\
\hline P13 & .777 \\
\hline P14 & .740 \\
\hline P15 & .772 \\
\hline P16 & .780 \\
\hline P17 & .778 \\
\hline
\end{tabular}

Elaboración propia

\section{Fiabilidad}

La medida más exacta utilizada para el diagnóstico de la fiabilidad es el coeficiente de Alpha de Cronbach, el cual valora la consistencia interna de la escala completa. En la Tabla 5 se muestra la fiabilidad interna (consistencia interna) de la escala total de los 3 factores (actividad física, estilos de vida y tiempo libre) que integran la investigación. La escala total muestra una Alpha de Cronbach de .780 considerando los 17 ítems del cuestionario. Cabe mencionar que los niveles de consistencia interna en otros estudios sobre la actividad física señalan una fiabilidad .059 y .064. ${ }^{(15)}$. Por otro lado, otros autores mencionan que el Coeficiente de Alpha de Cronbach depende del número de ítems, esto se refiere que en la medida en que este aumenta lo hace también su consistencia interna ${ }^{(16)}$.

El coeficiente de Guttman de dos mitades es de .751, por lo cual, la estructura interna es adecuada para este estudio. Para establecer la confiabilidad es recomendado el usó del coeficiente de Spearman-Brown y Guttman ${ }^{(17) .}$

Tabla N॰ 05

Fiabilidad del cuestionario

\begin{tabular}{|c|c|}
\hline COEFICIENTE & RESULTADO \\
\hline Coeficiente de Alpha de Cronbach & .780 \\
\hline Coeficiente de Guttman & .751 \\
\hline
\end{tabular}

\section{Elaboración propia}

El resultado de la validez del instrumento es congruente con el estudio de Leyton y Col donde encontraron consistencia interna con el Coeficiente Alpha de Cronbach .850 en un instrumento que validaba el estilo de vida saludable en españoles ${ }^{(18)}$. Al igual que en el estudio de García y Col que obtuvieron una fiabilidad del Alpha de Cronbach de .974 en su cuestionario para valorar escolares de Valladolid ${ }^{(19)}$. Las principales limitaciones que se encontraron en el instrumento fueron que los instrumentos validados para evaluar el ejercicio físico o las conductas sedentarias no corresponden al estilo de vida de los universitarios, además de la complejidad de las disciplinas que conforman la Facultad de Enfermería de la UAQ, ya que, a pesar de ser carreras relacionadas al área de la salud, dichos programas no promueven la práctica del ejercicio físico de manera regular.

\section{Conclusiones}

El estudio en universitarios aporta conocimientos sobre sus estilos de vida, caracterizada por largas jornadas académicas y actividades fuera de la institución, como las prácticas profesionales, el servicio social y tareas. Además, de contar con poco tiempo de descanso y 
por tanto insuficiente para realizar actividad física de manera regular, la mayoría de los universitarios consideran tener un estilo de vida sedentario. Es de suma importancia destacar que a pesar de que los estudiantes pertenecen a carreras relacionadas a la salud, a medida que avanzan los semestres, disminuye la cantidad efectiva de actividad física, mientras de forma inversa, incrementa el uso del tiempo libre destinado a redes sociales y medios como la televisión.

El contraste de los resultados obtenidos en ambas fases da evidencia de una satisfactoria materialización de los datos discursivos y sus indicadores en los ítems y resultados del instrumento cuantitativo. En suma, se ofrece una propuesta de instrumento de recolección de datos objetiva y fiable, pero que también atiende las características más relevantes y esenciales de los programas educativos para los cuales fue diseñado. De tal forma, se entiende que el cuestionario propuesto en este estudio es adecuado para estimar de manera objetiva y contextualizada conductas y hábitos en estudiantes universitarios queretanos.

\section{Referencias}

1. Guthold R, Stevens GA, Riley LM, Bull FC. Global trends in insufficient physical activity among adolescents: a pooled analysis of 298 population-based surveys with 1.6 million participants. Lancet Child Adolesc Health [Internet]. 2020 [Consultada 19 Nov 2021]; 4(1): 23-35. Disponible en: https://www.sciencedirect.com/science/article/pii/S2352464219303232

2. Organización Mundial de la Salud. Recomendaciones mundiales sobre actividad física para la salud. Ginebra, Organización Mundial de la Salud [Internet]. Ginebra: Suiza, 2010 [Consultada 19 Nov 2021]. Disponible en: http://apps.who.int/iris/bitstream/handle/10665/44441/9789243599977_spa.pdf;jsessionid=616F D55A94EAD7160BD60DCF5C9515D5?sequence $=1$

3. Instituto Nacional de Estadística, Geografía e Informática. Estadísticas a propósito del día internacional del deporte para el desarrollo de la paz. Comunicado de prensa Núm 197/21 5 de abril de 2021. [Internet]. 2021. Ciudad de México, México. [Consultada 22 Sept 2021]. Disponible en: https://www.inegi.org.mx/contenidos/saladeprensa/aproposito/2021/EAPDeporte21.pdf

4. Silva JM, Méndez JC, Fernández JU. Determinantes sociodemográficas y nivel de actividad física en adolescentes del municipio de Querétaro. Rev. Sup Acade [Internet]. 2019 [Consultada 22 Sept 2021]; 52(1): 10-21. Disponible en: http://www.supauaq.org/images/sampledata/sup_academica_52_comprimido.pdf

5. Wei Min L, Gutiérrez H. Efectividad del cuestionario global e internacional de actividad física comparado con evaluaciones prácticas. Rev. Cub. Inv. Biom. [Internet]. 2020 [Consultada 19 Nov 2021]; 39(2): 1-19. Disponible en: http://scielo.sld.cu/pdf/ibi/v39n2/1561-3011-ibi-39-02-e410.pdf

6. Arango EF, Echavarría AM, Aguilar FA, Patiño FA. Validación de dos cuestionarios para evaluar el nivel de actividad física y el tiempo sedentario en una comunidad universitaria de Colombia. Revista Facultad Nacional de Salud Pública [Internet]. 2020 [Consultada 17 Nov 2021]; 38(1): 1-11. Disponible en: http://www.scielo.org.co/pdf/rfnsp/v38n1/2256-3334-rfnsp-38-01-e334156.pdf

7. Barkley JE, Lepp A, Salehi-Esfahani S. College students' mobile telephone use is positively associated with sedentary behavior. Am J Lifestyle Med. [Internet]. 2016 [Consultada 17 Nov 2021]; 10(6): 437-441. Disponible en: https://pubmed.ncbi.nlm.nih.gov/30202304/

8. Peterson NE, Sirard JR, Kulbok PA, DeBoer MD, Erickson JM. Sedentary behavior and physical activity of young adult university students. Res Nurs Health. [Internet]. 2018 [Consultada 17 Nov 2021]; 41(1): 30-38. Disponible de: https://onlinelibrary.wiley.com/doi/epdf/10.1002/nur.21845

9. Hernández R, Mendoza C. Metodología de la Investigación: las rutas cuantitativa, cualitativa y mixta. Ed. Ciudad de México: México. Ed. McGraw Hill. 2018.

10. Organización Mundial de la Salud. The IPAQ group. International physical activity questionnaire [Internet]. 2016 [Consultada 18 Nov 2021]. https://sites.google.com/site/theipaq/

11. Organización Mundial de la Salud. Departamento de Enfermedades crónicas y Promoción de la Salud, Vigilancia y Prevención basada en la población. Cuestionario Mundial sobre Actividad Física (GPAQ) en español [Internet] Ginebra, Suiza. OMS; 2016 [Consultada 18 Nov 2021]. Disponible en: https://www.who.int/ncds/surveillance/steps/GPAQ_ES.pdf 
12. Carrillo-Mora P, Ramírez-Peris J, Magaña-Vázquez K. Neurobiología del sueño y su importancia: antología para el estudiante universitario. Rev. Fac. Med. UNAM. [Internet]. 2013 [Consultada 16 Nov 2021]; 56(4): 7-15. Disponible en: http://www.scielo.org.mx/pdf/facmed/v56n4/v56n4a2.pdf

13. Chales-Aoun AG, Merino Escobar JM. Physical activity and eating behaviors among chilean university students. Cienc Enferm [Internet]. 2019 [Consultada 16 Nov 2021]; 25(16): 1-10. Disponible en: https://scielo.conicyt.cl/pdf/cienf/v25/0717-9553-cienf-25-16.pdf

14. Práxedes A, Sevil J, Moreno A, del Villar F, García L. Niveles de actividad física y motivación en estudiantes universitarios. Diferencias en función del perfil académico vinculado a la práctica físico deportiva. Journal of Sport and Health Research [Internet]. 2016 [Consultada 17 Nov 2021]; 13(3): 191-204. Disponible en: http://www.journalshr.com/papers/Vol\%208_N\%203/V08_3_3.pdf

15. Hopman-Rock M, Dusseldorp E, Chorus A, Jacobusse G, Ruetten A, van Buuren S. Response conversion for improving comparability of international physical activity data. J Phys Act Health [Internet]. 2012 [Consultada 18 Nov 2021]; 9(1): 29-38. Disponible en: https://www.researchgate.net/publication/221735956_Response_Conversion_for_Improving_Com parability_of_International_Physical_Activity_Data

16. Orozco LC. Confiabilidad de la consistencia, reproducibilidad, acuerdo y algo más. En: OrozcoVargas LC, editor. Medición en salud. Diagnóstico y evaluación de resultados: un manual critico más allá de lo básico. Bucaramanga: Colombia. Ed. Publicaciones UIS; 2010; 73-103.

17. Guttman L. Fundamentos del análisis del escalo- grama. En: Wainerman $\mathrm{CH}$ (comp). Escalas de medición en ciencias sociales. 1ra Ed. Buenos Aires: Argentina. Ed. Ediciones Nueva Visión, 1976; 291-330.

18. Leyton M, Lobato S, Batista M, Aspano MI, Jiménez R. Validación del Cuestionario de Estilo de Vida Saludable (EVS) en una población española. Rev. Ibero. Psico. Ejer. Dep. [Internet]. 2016 [Consultada 18 Nov 2021]; 13(1): 23-31. Disponible en: https://www.redalyc.org/pdf/3111/311153534002.pdf

19. García IA, Muñoz MF, Ruíz G, Gil Blanca. Validación de un cuestionario sobre actitudes y práctica de actividad física y otros hábitos saludables mediante el método Delphi. Rev. Esp. Sal, Pub. [Internet]. 2019 [Consultada 18 Nov 2021]; 93: 16. Disponible en: https://scielo.isciii.es/pdf/resp/v93/11355727-resp-93-e201909081.pdf

\section{Editor}

Escuela de Enfermería de la Universidad Católica Santo Toribio de Mogrovejo, Chiclayo, Perú

\section{Cómo citar este trabajo}

Méndez-Ávila J, Silva-Llaca J, Ornelas-Murrieta A, Mendoza-Ayala M, Morales-Hernández A. Validación de un instrumento para determinar conductas sedentarias en universitarios. Acc Cietna: para el cuidado de la salud [Internet]. 2021; 8(2): 45 - 53. Disponible en: https://doi.org/10.35383/cietna.v8i2.652

\section{Financiación}

El presente artículo no cuenta con financiación específica de agencias de financiamiento en los sectores público o privado para su desarrollo y/o publicación.

\section{Conflicto de interés}

Los autores del artículo declaran no tener ningún conflicto de intereses en su realización.

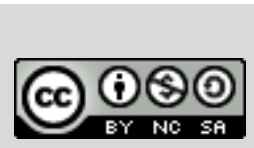

(c) Los autores. Este artículo es publicado por la Revista Acc Cietna: para el cuidado de la salud de la Escuela de Enfermería, Universidad Católica Santo Toribio de Mogrovejo.

Este es un artículo de acceso abierto, distribuido bajo los términos de la Licencia Creative Commons Atribución-NoComercial-CompartirIgual 4.0 Internacional (CC BY-NC-SA 4.0), que permite el uso no comercial, distribución y reproducción en cualquier medio, siempre que la obra original sea debidamente citada. 